\title{
NADPH oxidase 3-associated oxidative stress and caspase 3-dependent apoptosis in the cochleae of D-galactose-induced aged rats
}

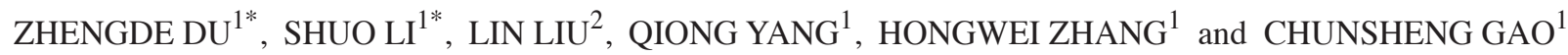 \\ ${ }^{1}$ Department of Otorhinolaryngology, Nanshan Affiliated Hospital of Guangdong Medical College, \\ Shenzhen, Guangdong 518052; ${ }^{2}$ Department of Pharmacology, College of Pharmacy, Guangxi \\ Medical University, Nanning, Guangxi Zhuang Autonomous Region 530021, P.R. China
}

Received October 21, 2014; Accepted July 23, 2015

DOI: $10.3892 / \mathrm{mmr} .2015 .4430$

\begin{abstract}
Oxidative damage to mitochondrial DNA (mtDNA) and cell apoptosis are heavily implicated in aging. Our previous study established a mimetic rat model of aging in the cochleae using D-galactose (D-gal), and revealed that chronic injection of D-gal can increase oxidative stress and mtDNA common deletions (CD). The aim of the present study was to investigate the sources of reactive oxygen species and the occurrence of apoptosis in the cochleae of rats following 8 weeks of D-gal exposure. The results of the present study indicated that an elevated accumulation of the mtDNA CD and mitochondrial ultrastructural damage occurred in the cochleae of rats injected with D-gal for 8 weeks. In addition, the levels of 8-hydroxy-2-deoxyguanosine, NADPH oxidase (NOX) 3, P22 $2^{\text {phox }}$ and cleaved caspase 3, and the number of terminal deoxynucleotidyl transferase-mediated deoxyuridine triphosphate nick-end-labelling-positive cells were increased in the cochleae of D-gal-treated rats, compared with the controls. These findings suggested that nitric oxide
\end{abstract}

Correspondence to: Dr Chunsheng Gao, Department of Otorhinolaryngology, Nanshan Affiliated Hospital of Guangdong Medical College, 89 Taoyuan Road, Shenzhen, Guangdong 518052, P.R. China

E-mail: entcsgao@163.com

${ }^{*}$ Contributed equally

Abbreviations: ABR, auditory brainstem response; C-cas3, cleaved caspase 3; CD, common deletion; D-gal, D-galactose; MDA, malondialdehyde; mtDNA, mitochondrial DNA; NOX3, NADPH oxidase 3; 8-OHdG, 8-hydroxy-2-deoxyguanosine; OC, organ of Corti; ROS, reactive oxygen species; SG, spiral ganglion; SGC, spiral ganglion cell; SV, stria vascularis; T-SOD, total superoxide dismutase; TUNEL, terminal deoxynucleotidyl transferase-mediated deoxyuridine triphosphate nick-end-labelling

Key words: age-related hearing loss, mitochondria, NADPH oxidase 3 , oxidative stress, cleaved caspase 3 , apoptosis synthase NOX3-associated oxidative stress may contribute to the accumulation of mtDNA mutations and activate a caspase 3-dependent apoptotic signalling pathway in the cochleae during aging. The present study also provided novel insights into the development of age-associated hearing loss, also termed presbycusis.

\section{Introduction}

Age-associated hearing loss, also known as presbycusis, is characterised by an age-dependent decline of auditory function associated with loss of sensory hair cells, spiral ganglion neurons and stria vascularis cells in the cochleae of the inner ear $(1,2)$. However, the exact pathogenesis of age-related hearing loss remains to be elucidated.

As the cochleae tissue is not acquirable from humans during life, and the genetic and environmental background of individuals with hearing loss is heterogenous, the investigation of presbycusis is relatively limited. Natural aging can be experimentally modelled by the chronic administration of D-galactose (D-gal). Animals treated in this way exhibit a reduction in the activity of antioxidant enzymes (3-5), dysfunctional mitochondria $(6-8)$, increased apoptosis $(9,10)$ and neurotoxicity $(7,11,12)$. Consequently, these animals exhibit a shortened lifespan (13), poor learning and memory (14-16) and an attenuated immune response (17-19). These characteristics are considered to be associated with an increase in oxidative stress caused by a metabolic disturbance. Previous studies have established a mimetic aging model in the cochleae of rats following 8 weeks of D-gal treatment, and demonstrated that the activity levels of antioxidant enzymes decreased and those of lipid peroxidation increased in this model (20-22). Furthermore, the levels of mitochondrial DNA (mtDNA) common deletion (CD) were significantly increased in the cochleae of the D-gal-treated rats (20-24). However, the sources of reactive oxygen species (ROS) and the effects of mtDNA CD in the cochleae of rats from this model remain to be fully elucidated.

In addition to mitochondria, the NADPH oxidase (NOX) system is one of the predominant ROS-generating sites, and it is now clear that NOX is not restricted to the immune system, 
and that alternative isoforms may be active in several other cell types as essential components of cellular signalling, gene expression regulation and cell differentiation (25). These enzymes share the capacity to transport electrons across the plasma membrane and to generate superoxide and other downstream ROS (25). A previous study reported that the expression levels of NOX3 are higher in the cochleae than in any other tissue (26). NOX3 forms a functional complex with P22 $2^{\text {phox }}$ to produce superoxide (27). Previous studies have demonstrated that NOX3 is a relevant source of ROS generation in the cochleae, and that NOX3-dependent ROS generation may contribute to hearing loss in response to ototoxic drugs $(26,28-30)$.

Apoptosis may be important in the age-related decline of physiological function in several organs (31), including aging in the cochleae $(32,33)$. A previous investigation demonstrated that D-gal-induced apoptotic cells are significantly increased in the cochlear section of newborn rats (34). Previous studies have also reported that apoptotic cells immediately increase in the central auditory system of adult rats following 8 weeks of treatment with D-gal $(35,36)$. Du et al $(37)$ reported that apoptotic cells increase in the peripheral auditory system of D-gal-treated aging rats following 12 months of treatment. However, whether 8 weeks of treatment with D-gal immediately causes apoptosis in the cochleae of adult rats has not been investigated. In the present study, the accumulation of mtDNA CD, mitochondrial ultrastructural changes and changes in the expression levels of 8-OHdG, NOX3, P22 $2^{\text {phox }}$ and cleaved caspase 3 (C-cas3) were investigated, as well as the occurrence of apoptosis in the cochleae of rats exposed to D-gal for 8 weeks. Furthermore, the present study also investigated the possible mechanism underlying presbycusis using D-gal-induced aging rats.

\section{Materials and methods}

Animals and treatments. A total of 601 month old male Sprague-Dawley rats were obtained from the Experimental Animal Centre of the Guangxi Medical University (Guangxi, China). The rats were individually housed in a temperature-controlled $\left(20-22^{\circ} \mathrm{C}\right)$ room with a $12 \mathrm{~h}$ light/dark cycle, and were provided with free access to food and drinking water. The body weights of the experimental animals were monitored during the experiment as a general measure of health. The injection of D-gal (Sigma-Aldrich, St. Louis, MO, USA) to induce aging was administered, according to an established method (37). Following acclimation for 2 weeks, the rats were randomly divided into three groups: (1) D-gal(H) group, injected subcutaneously with $500 \mathrm{mg} / \mathrm{kg}$ D-gal once a day for 8 weeks; (2) D-gal(L) group, injected subcutaneously with $150 \mathrm{mg} / \mathrm{kg}$ D-gal once a day for 8 weeks; (3) control group, which were administered with an equal volume of vehicle ( $0.9 \%$ saline) for 8 weeks. Following the experimentation period, the rats were anaesthetised with intraperitoneally injected ketamine $(30 \mathrm{mg} / \mathrm{kg}$; Maijin Biotechnology, Hubei, China) and intramuscular injected chloropromazine (15 mg/kg; Maijin Biotechnology), and blood samples $(6 \mathrm{ml} / \mathrm{rat})$ were obtained from the heart. Serum was obtained by centrifugation at $800 \mathrm{x} \mathrm{g}$ for $15 \mathrm{~min}$ at $4^{\circ} \mathrm{C}$, and stored at $-80^{\circ} \mathrm{C}$ until the assessments of $\mathrm{H}_{2} \mathrm{O}_{2}$, total superoxide dismutase (T-SOD) activity and malondialdehyde (MDA) levels were performed. The cochleae were dissected and used for the extraction of total RNA, genomic DNA and protein. Alternatively, the cochleae were perfused with 2.5\% glutaraldehyde (Maijin Biotechnology) for morphological investigation using transmission electron microscopy (TEM), or with 4\% paraformaldehyde (Maijin Biotechnology) for immunohistochemical analysis and terminal deoxynucleotidyl transferase-mediated deoxyuridine triphosphate nick-end-labelling (TUNEL) staining. All experiments were conducted in strict accordance with the recommendations in the Guide for the Care and Use of Laboratory Animals of the National Institutes of Health. The protocol was approved by the Committee on the Ethics of Animal Experiments of Guangxi Medical University.

Serum $\mathrm{H}_{2} \mathrm{O}_{2}, T-S O D$ activity and MDA assays. Using the serum from 30 rats ( $n=10$ per group), the levels of $\mathrm{H}_{2} \mathrm{O}_{2}, \mathrm{~T}-\mathrm{SOD}$ activity and MDA were quantified using $\mathrm{H}_{2} \mathrm{O}_{2}$ Assay, T-SOD Assay and MDA Assay kits, respectively (Nanjing Jiancheng Chemical Industrial Co., Ltd, Nanjing, China), according to the manufacturer's instructions.

DNA isolation and determination of mtDNA CD. Following the final injection, 18 rats ( $\mathrm{n}=6$ per group) were euthanised under deep anaesthesia with chlorpromazine $(15 \mathrm{mg} / \mathrm{kg}$; Maijin Biotechnology) and ketamine hydrochloride $(30 \mathrm{mg} / \mathrm{kg}$; Maijin Biotechnology), and the cochlea from both sides of each rat were rapidly removed. The soft tissue samples were then harvested from the cochleae using an anatomical microscope (Nikon Corporation, Tokyo, Japan). Samples were stored at $-80^{\circ} \mathrm{C}$ until experimentation. The cochlea from one side was used for mtDNA analysis and that from the other side was used for RNA extraction. Total DNA was extracted using a Genomic DNA Purification kit (Tiangen Biotech Co., Ltd, Beijing, China), according to the manufacturer's instructions. The DNA concentration of each specimen was measured using a GeneQuant pro DNA/RNA Calculator (BioChrom, Cambridge, UK). The quantity of the mtDNA CD was determined using a TaqMan polymerase chain reaction (PCR) assay kit (Takara Biotechnology Co., Ltd., Dalian, China). Due to the fact that the D-Loop region is rarely deleted, it can represent the conserved segment. Primers and probes for the mtDNA D-loop and the mtDNA CD have previously been described (38), and were as follows: Forward, 5'-GGTTCTTACTTCAGGGCCATCA-3'; reverse, 5'-GATTAGACCCGTTACCATCGAGAT-3' for the mtDNA D-loop primers and 5'-FAM-TTGGTTCATCGTCCATACG TTCCCCTTA-TAMRA-3' for the probe; and forward, 5'-AAGGACGAACCTGAGCCCTAATA-3'; reverse, 5'-CGAAGTAGATGATCCGTATGCTGTA-3' for the mIDNA CD primers and 5'-FAM-TCACTTTAATCGCCAC ATCCATAACTGCTGT-TAMRA-3' for the probe. The PCR amplification was performed on a StepOnePlus ${ }^{\mathrm{TM}}$ Real-Time PCR system (Applied Biosystems Life Technologies, Foster City, CA, USA) in a $20 \mu \mathrm{l}$ reaction volume consisting of $10 \mu \mathrm{l}$ 2X TaqMan PCR mix (Takara Biotechnology Co., Ltd.), $0.4 \mu \mathrm{l}$ 50X ROX reference dye, $0.4 \mu \mathrm{l}$ of each forward and reverse primer $(10 \mu \mathrm{M}), 0.2 \mu \mathrm{l}$ of each probe $(10 \mu \mathrm{M}), 4 \mu \mathrm{l}$ of the sample DNA (10 ng/ $/ \mu$ ) and $4.6 \mu 1$ distilled water. 
The cycling conditions comprised an initial phase at $95^{\circ} \mathrm{C}$ for $30 \mathrm{sec}$, followed by 40 cycles at $95^{\circ} \mathrm{C}$ for $5 \mathrm{sec}$ and at $60^{\circ} \mathrm{C}$ for $30 \mathrm{sec}$. The cycle number at which a significant increase in the normalised fluorescence was first detected was designated as the threshold cycle $(\mathrm{Ct})$. The ratio of mtDNA CD to mtDNA was calculated using the following equation: $\Delta \mathrm{Ct}=\mathrm{Ct}_{\mathrm{mtDNA} \text { deletion }}-\mathrm{Ct}_{\mathrm{mtDNA} \text { D-loop }}$. The relative expression (RE) was calculated to indicate the factorial difference in the deletions between the experimental groups and the control group. The RE was calculated using

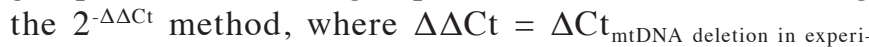
mental group $-\Delta \mathrm{CtmtDNA}_{\text {deletion in control group }}$.

TEM. The ultrastructure of the mitochondria in the spiral ganglion cell (SGC) of the cochleae was observed using TEM. A total of 12 rats ( $n=4$ per group) were sacrificed, and both cochleae from each rat were removed, treated with $2.5 \%$ glutaraldehyde and fixed overnight at $4^{\circ} \mathrm{C}$. The following day, the cochleae were washed with $0.1 \mathrm{M}$ phosphate-buffered saline (PBS) and placed in 10\% ethylenediaminetetraacetic acid solution (EDTA; Maijin Biotechnology) for decalcification for 3 days. The spiral ganglion (SG) was carefully dissected and harvested from the cochleae using an anatomical microscope. Following post-fixation in $1 \%$ osmium tetroxide (Maijin Biotechnology) for $2 \mathrm{~h}$ at room temperature, the SG was dehydrated using graded ethanol or acetone $(50,70,80$, 90 and $100 \%$ ), immersed in an acetone/Epon 812 mixture (1:1) for $2 \mathrm{~h}$, followed by immersion in Epon 812 for $2 \mathrm{~h}$ and final embedding in Epon 812 for $10 \mathrm{~h}$ at $80^{\circ} \mathrm{C}$. Serial ultrathin sections $(50 \mathrm{~nm})$ were collected on copper grids and stained with uranyl acetate and lead citrate. The ultrastructure of the stained sections were examined using a FEI TecnaiG ${ }^{2} 12$ TEM (Philips, Amsterdam, Netherlands).

$R N A$ preparation and reverse transcription-quantitative $(R T-q)$ $P C R$. The mRNA expression levels of NOX3 and P22 $2^{\text {phox }}$ were determined using RT-qPCR. Total RNA was extracted using TRIzol $^{\circledR}$ reagent (Takara Biotechnology Co., Ltd.), according to the manufacturer's instructions. cDNA was reverse transcribed using a PrimeScript RT reagent kit (Takara Biotechnology Co., Ltd.). The RNA and cDNA of each sample were analysed using a GeneQuant pro DNA/RNA calculator to assess the concentrations and purity. The cDNA samples (n=6/group) were stored at $-20^{\circ} \mathrm{C}$ until further use. RT-qPCR was performed using real-time SYBR Green PCR technology with a StepOnePlus ${ }^{\mathrm{TM}}$ Real-Time PCR system (Applied Biosystems Life Technologies). The primer pairs for NOX3,P22 $2^{\text {phox }}$ and the internal standard ( $\beta$-actin) were as follows: NOX3, forward 5'-TCGACGAATGGCAGGAAGC-3' and reverse 5'-ATGGATGGGCACTGGATAAAG-3'; P22 ${ }^{\text {phox }}$, forward 5'-ACCGTCTGCTTGGCCATTG-3' and reverse 5'-TCAATGGGAGTCCACTGCTCAC-3'; and $\beta$-actin, forward 5'-CCTGGAGAAGAGCTATGAGC-3'; and reverse 5'-ACAGGATTCCATACCCAGG-3'. The amplification thermocycling conditions were as follows: $30 \mathrm{sec}$ at $95^{\circ} \mathrm{C}, 40$ cycles of $5 \mathrm{sec}$ at $95^{\circ} \mathrm{C}, 30 \mathrm{sec}$ at $60^{\circ} \mathrm{C}$ and $30 \mathrm{sec}$ at $72^{\circ} \mathrm{C}$. An internal standard was used to normalise the relative gene expression levels. Subsequent melting curve analysis was performed for each gene, and the specificity and integrity of the PCR products were confirmed by the presence of a single peak. The relative expression levels were calculated from the variations in
$\mathrm{Ct}$ values between the target mRNA and the internal standard ( $\beta$-actin). Changes in the relative mRNA expression levels between the experimental and control groups were analysed using the $2^{-\Delta \Delta C t}$ method, as previously reported (39).

Immunohistochemical analysis. The expression of 8-hydroxy-2-deoxyguanosine (8-OHdG) expression was analysed using immunohistochemistry. A total of 12 rats $(n=4$ per group) were sacrificed, and the cochleae from each rat removed and fixed with $4 \%$ buffered-paraformaldehyde overnight, followed by decalcification with $10 \%$ EDTA in PBS for 2 weeks, dehydration and embedding in paraffin wax. The cochlea from one side was used for immunohistochemical analysis, and the cochlea from the other side was used for the TUNEL assay. A $5 \mu \mathrm{m}$ section was deparaffinised in xylene and rehydrated through graded concentrations of ethanol. The samples were incubated with mouse monoclonal anti-8-OHdG antibody (1:4,000; Abcam, Cambridge, MA, USA) overnight at $4^{\circ} \mathrm{C}$. The samples were then incubated with CY3-labelled goat anti-mouse secondary antibody (1:200; Wuhan Boster Biological Technology, Ltd., Wuhan, China) for $30 \mathrm{~min}$ at room temperature. The nuclei were counterstained with DAPI staining solution (Beyotime Institute of Biotechnology, Haimen, China) for $5 \mathrm{~min}$ at room temperature. For immunofluorescence imaging, the slides were visualised using a laser scanning confocal microscope (Nikon Corporation, Tokyo, Japan) and analysed using Image-Pro Plus 6.0 software (Media Cybernetics, Inc., Rockville, MD, USA).

Western blot analysis. The protein expression levels of NOX $3, \mathrm{P} 22^{\text {phox }}$ and C-cas3 were determined using western blot analysis. A total of 18 rats ( $n=6$ per group) were sacrificed, and soft tissue samples $(\sim 500 \mu \mathrm{g})$ of the cochleae from each rat were dissected. Total protein was extracted using Radioimmunoprecipitation Assay Lysis buffer (Beyotime Institute of Biotechnology), according to the manufacturer's instructions. Protein concentrations were determined using an Enhanced Bicinchoninic Acid Protein assay kit (Beyotime Institute of Biotechnology). A total of $30 \mu \mathrm{g}$ of each protein lysate was separated by $12 \%$ SDS-PAGE (Maijin Biotechnology) and transferred onto polyvinylidene difluoride membranes (Maijin Biotechnology). The membranes were incubated for $1 \mathrm{~h}$ in a blocking solution, Tris-buffered saline (TBS; Maijin Biotechnology) containing 5\% skimmed milk, and then washed briefly in TBS. The membranes were subsequently incubated overnight at $4^{\circ} \mathrm{C}$ with the appropriate dilution of rabbit polyclonal anti-NOX3 (1:200; Santa Cruz Biotechnology, Inc., Dallas, TX, USA), rabbit polyclonal anti-p22 $2^{\text {phox }}$ (1:100; Wuhan Boster Biological Technology, Ltd.) and rabbit monoclonal anti-C-cas3 (1:1,000; Cell Signaling Technology, Inc., Danvers, MA, USA) antibodies. Following membrane washing with TBS, to remove excess primary antibody, the membranes were incubated for $1 \mathrm{~h}$ at room temperature with the appropriate horseradish peroxidase-conjugated goat anti-rabbit secondary antibody (1:5,000; Santa Cruz Biotechnology, Inc.). The membranes were visualised using BeyoECL Plus (Beyotime Institute of Biotechnology). Quantification of the detected bands was performed using Image-Pro Plus 6.0 software. $\beta$-actin served as an internal control. 
Table I. Serum levels of $\mathrm{H}_{2} \mathrm{O}_{2}$, T-SOD activity and MDA following treatment with D-gal.

\begin{tabular}{lrrr}
\hline Compound & Control & D-gal $(\mathrm{L})$ & D-gal $(\mathrm{H})$ \\
\hline $\mathrm{H}_{2} \mathrm{O}_{2}(\mu \mathrm{mol} / \mathrm{ml})$ & $14.65 \pm 1.78$ & $19.37 \pm 1.82^{\mathrm{a}}$ & $27.88 \pm 3.31^{\mathrm{a}}$ \\
$\mathrm{T}-\mathrm{SOD}(\mathrm{U} / \mathrm{ml})$ & $146.99 \pm 7.10$ & $118.11 \pm 3.95^{\mathrm{a}}$ & $97.59 \pm 3.81^{\mathrm{a}}$ \\
$\mathrm{MDA}(\mathrm{nmol} / \mathrm{ml})$ & $2.44 \pm 0.56$ & $5.04 \pm 0.93^{\mathrm{a}}$ & $7.65 \pm 1.09^{\mathrm{a}}$ \\
\hline
\end{tabular}

Data are expressed as means \pm standard deviation (10 rats per group). ${ }^{\mathrm{a}} \mathrm{P}<0.01$, vs. control group. D-gal, D-galactose; T-SOD, total superoxide dismutase; MDA, malondialdehyde; H, $500 \mathrm{mg} / \mathrm{kg} ; \mathrm{L}, 150 \mathrm{mg} / \mathrm{kg}$.

TUNEL assay. Apoptotic cells were detected in situ using a TUNEL POD assay kit (Roche Diagnostics GmbH, Mannheim, Germany). Briefly, the tissue sections were deparaffinized through a concentration gradient of xylene and rehydrated with distilled water. Following treatment with proteinase $\mathrm{K}$ (20 $\mu \mathrm{g} / \mathrm{ml}$; Beyotime Institute of Biotechnology) in $10 \mathrm{mM}$ Tris- $\mathrm{HCl}(\mathrm{pH} 7.6)$ for $10 \mathrm{~min}$ at $37^{\circ} \mathrm{C}$, the sections were washed in PBS, and the labelling reaction was performed using labelling solution containing terminal deoxynucleotidyl transferase, its buffer, and fluorescein $\mathrm{dUTP}$ at $37^{\circ} \mathrm{C}$ for $60 \mathrm{~min}$ in a humidity chamber. The nuclei were counterstained using DAPI staining solution for $5 \mathrm{~min}$ at room temperature. Following washing with PBS, the sections were examined using a laser scanning confocal microscope (C1si; Nikon Corporation, Tokyo, Japan).

Statistical analysis. The data are presented as the mean \pm standard deviation. Statistical significance was determined using a one-way analysis of variance, and a least significant difference post-hoc test was used to evaluate the statistical differences between groups. Analyses were performed using SPSS 13.0 software (SPSS, Inc., Chicago, IL, USA). $\mathrm{P}<0.05$ was considered to indicate a statistically significant difference.

\section{Results}

Oxidative stress is induced by $D$-gal. The serum levels of $\mathrm{H}_{2} \mathrm{O}_{2}$, T-SOD and MDA from the rats are summarised in Table 1. Following 8 weeks D-gal exposure, the serum levels of $\mathrm{H}_{2} \mathrm{O}_{2}$ and MDA were significantly higher, and the serum activity levels of T-SOD were markedly lower, compared with the control group.

Age-associated accumulation of mtDNA CD is induced by $D$-gal. To evaluate the level of mtDNA damage induced by D-gal in the cochleae, the levels of mtDNA CD were determined using RT-qPCR with a TaqMan probe. The dual-labelled fluorescent DNA probe used was specific for the novel fusion sequence, which was present only in mutant mtDNA, which contained the CD. As shown in Fig. 1, the levels of mtDNA CD were significantly higher in the D-gal group, compared with the control group. Compared with the control group, the accumulation of mtDNA CD in the D-gal $(\mathrm{L})$ group and in the D-gal $(\mathrm{H})$ group were increased by 1.45 - and 2.23 -fold, respectively.

Mitochondrial ultrastructural damage is induced by D-gal. To further investigate the mitochondrial damage induced by

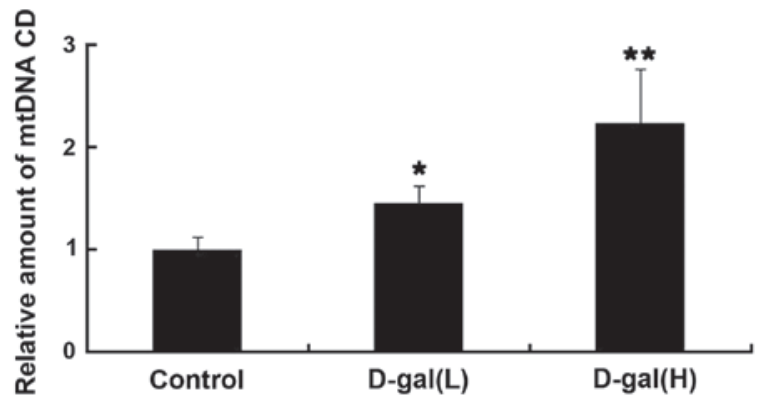

Figure 1. Quantitative analysis of the levels of mtDNA CD in the cochleae of rats from the control and D-gal groups. The levels of mtDNA CD were significantly higher in the D-gal groups, compared with the control group. The data are expressed as the mean \pm standard deviation of six rats per group. ${ }^{*} \mathrm{P}<0.05$ and ${ }^{* *} \mathrm{P}<0.01$, vs. control group. D-gal, D-galactose; mtDNA, mitochondrial DNA; CD, common deletion.

D-gal in the cochleae, changes to the mitochondrial ultrastructure in the SGC of the cochleae were observed using TEM. In the control group, numerous round and oval mitochondria with lamellar cristae were present, predominantly around the nucleus of the SGC (Fig. 2A). By contrast, the mitochondria in the SGC of the D-gal groups were swollen with reduced electron density in the matrix or exhibiting severe degeneration. Furthermore, the lipofuscins were also deposited in the SGC of the D-gal groups, indicating structural decay (Figs. 2B-C).

Oxidative mtDNA damage is induced by D-gal. To determine whether increased mtDNA CD was associated with increased oxidative stress induced by D-gal in the cochleae, the expression levels of 8-OHdG, a biomarker of DNA oxidative damage, were analysed using immunohistochemical analysis (Fig. 3). As shown in Fig. 3A, the expression levels of $8-\mathrm{OHdG}$ were markedly increased in the cytoplasm of the cochleae cells from the D-gal-induced aging rats, compared with those of the control rats, which suggested that D-gal increased oxidative mtDNA damage in the cochleae. Compared with the control group, the immunohistochemical analysis indicated that the expression levels of $8-\mathrm{OHdG}$ in the $\mathrm{D}$-gal $(\mathrm{L})$ and the $\mathrm{D}-\mathrm{gal}(\mathrm{H})$ groups increased by 3.24- and 6.59-fold, respectively (Fig. 3B).

Increased mRNA expression levels of NOX 3 and P22 phox are induced by $D$-gal. To investigate the effects of NOX3-associated oxidative stress on the mtDNA damage induced by D-gal in the cochleae, the mRNA expression levels of NOX3 and P22 $2^{\text {phox }}$ were determined using an RT-qPCR assay. As shown 
A

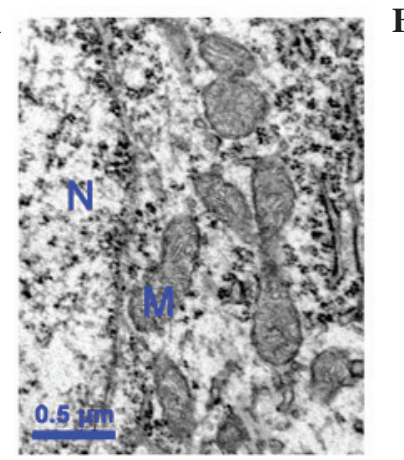

B

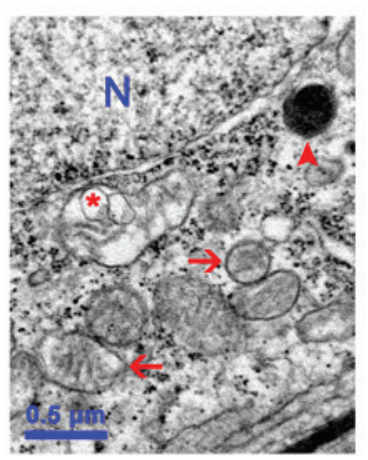

C

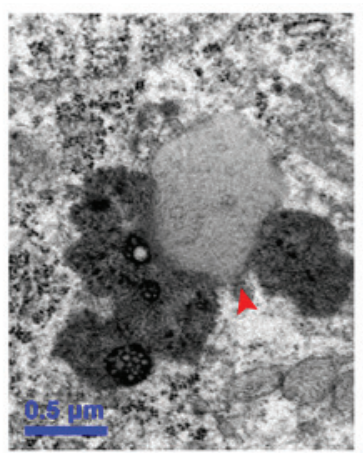

D

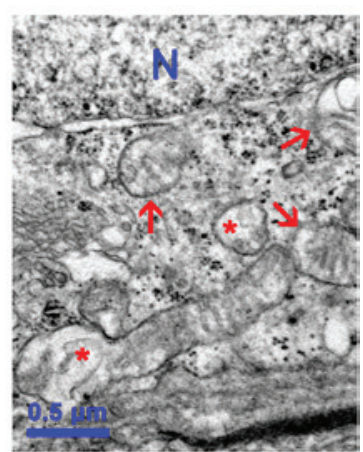

Figure 2. Changes in the mitochondrial ultrastructure of the SGC in the basal turn of the cochleae. (A) Normal mitochondria surrounding the nucleus of the SGC in the control group. (B) Mitochondria with reduced electron density (arrows) or severe degeneration (*) in the SGC of the D-gal(L) group. Lipofuscin (arrowhead) deposited in the SGC of the D-gal(L) group, indicating structural decay. (C) Lipofuscins (arrowhead) deposited in the SGC of the D-gal(H) group, indicating structural decay. (D) Mitochondria with reduced electron density (arrows) or severe degeneration (*) in the SGC of the D-gal(H) group. Scale bar=0.5 $\mu \mathrm{m}$. D-gal, D-galactose; H, $500 \mathrm{mg} / \mathrm{kg} ; \mathrm{L}, 150 \mathrm{mg} / \mathrm{kg} ; \mathrm{M}$, mitochondria; N, nucleus; SGC, spiral ganglion cell.

A
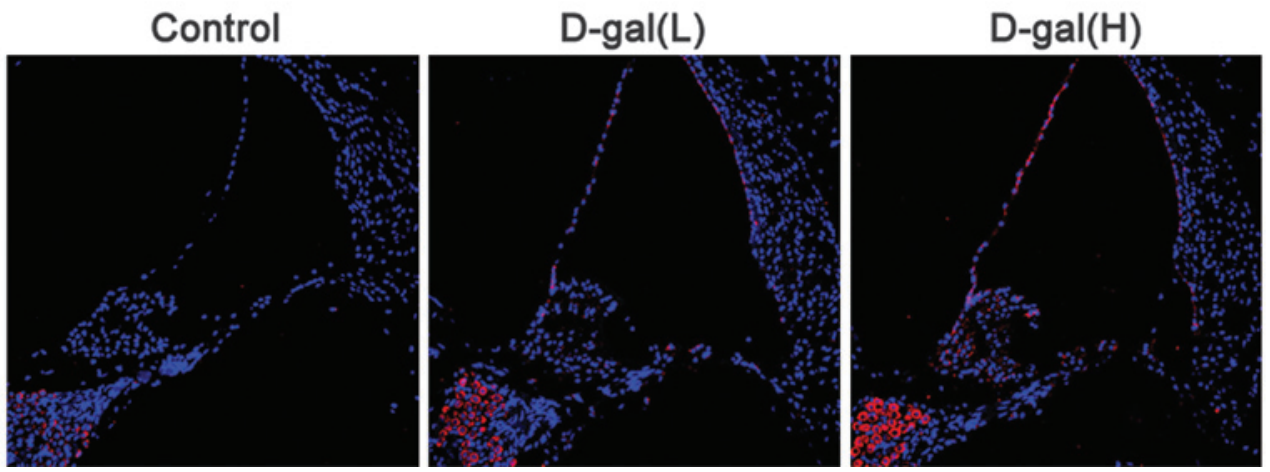

B

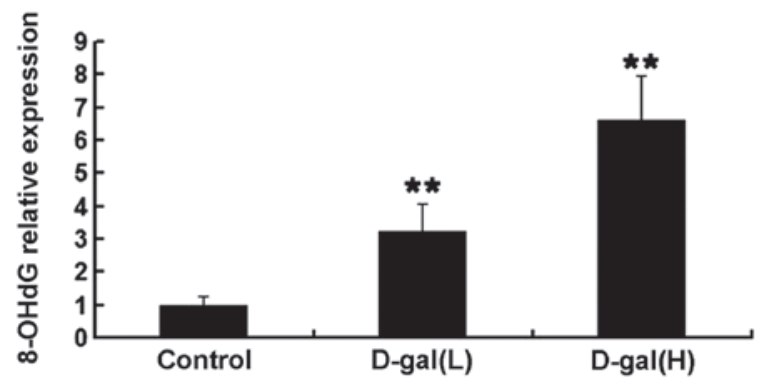

Figure 3. mtDNA oxidative damage, detected using 8-OHdG immunohistochemistry. (A) Expression and localization of 8-OHdG in the basal turn of the cochleae from the control and D-gal groups. The nuclei were labeled with DAPI. Fluorescent images of the cochleae are presented at $\mathrm{x} 200 \mathrm{magnification}$ (B) Quantification of the expression levels of 8-OHdG. The expression levels of 8-OHdG in the D-gal groups were significantly increased, compared with those in the control group. The data are expressed as the mean \pm standard deviation of four rats per group. ${ }^{* *} \mathrm{P}<0.01, \mathrm{vs}$. control group. $\mathrm{D}$-gal, $\mathrm{D}$-galactose; $\mathrm{H}$, $500 \mathrm{mg} / \mathrm{kg} ; \mathrm{L}, 150 \mathrm{mg} / \mathrm{kg}$; mtDNA, mitochondrial DNA; 8-OHdG, 8-hydroxy-2-deoxyguanosine.

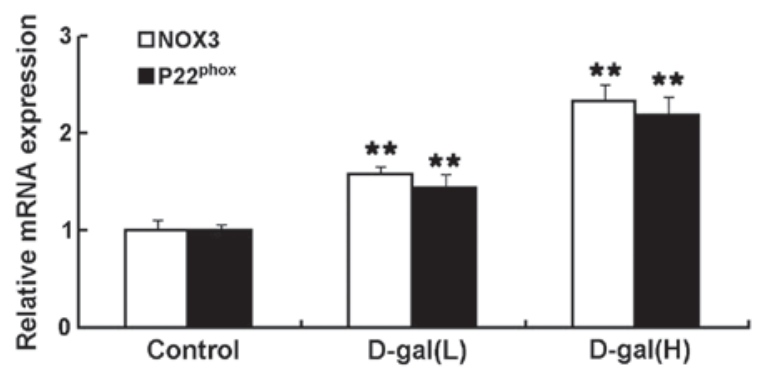

Figure 4. Quantitative analysis of the mRNA expression levels of NOX3 and $\mathrm{P} 22^{\text {phox }}$ in the cochleae of rats from the control and D-gal groups. The mRNA expression levels of NOX 3 and $\mathrm{P} 22^{\text {phox }}$ were significantly increased in the D-gal groups, compared with the control group. The data are expressed as the mean \pm standard deviation of six rats per group. ${ }^{* *} \mathrm{P}<0.01$, vs. control group. D-gal, D-galactose; H, 500 mg/kg; L, 150 mg/kg. in Fig. 4, the mRNA expression levels of NOX3 and P22 $2^{\text {phox }}$ were significantly higher in the D-gal groups, compared with the control group. Compared with the control group, the mRNA expression levels of NOX3 in the D-gal(L) and $\mathrm{D}$-gal $(\mathrm{H})$ group increased by 1.57 - and 2.33 -fold, respectively. The mRNA expression levels of P22 $2^{\text {phox }}$ in the D-gal $(\mathrm{L})$ and D-gal $(\mathrm{H})$ group increased by 1.43 - and 2.19 -fold, respectively.

Increased protein expression levels of NOX $3, P 22^{\text {phox }}$ and $C$-cas 3 are induced by $D$-gal. To examine the protein expression levels of NOX3, P22 $2^{\text {phox }}$ and C-cas 3 in the cochleae, western blot analysis was performed. As shown in Fig. 5A, the protein expression levels of NOX3, P22 $2^{\text {phox }}$ and C-cas3 were markedly increased following treatment with D-gal. 
A

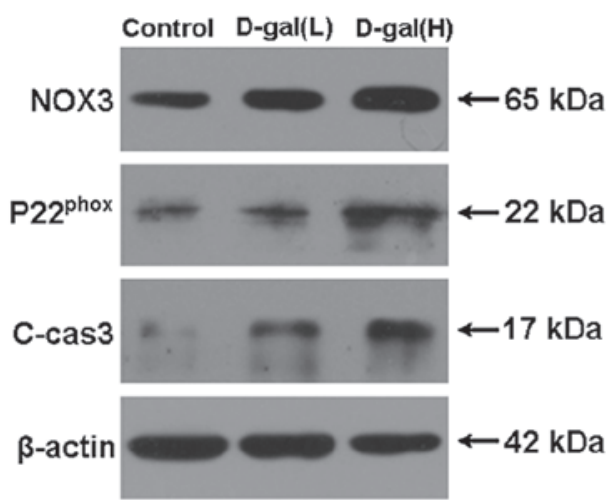

B

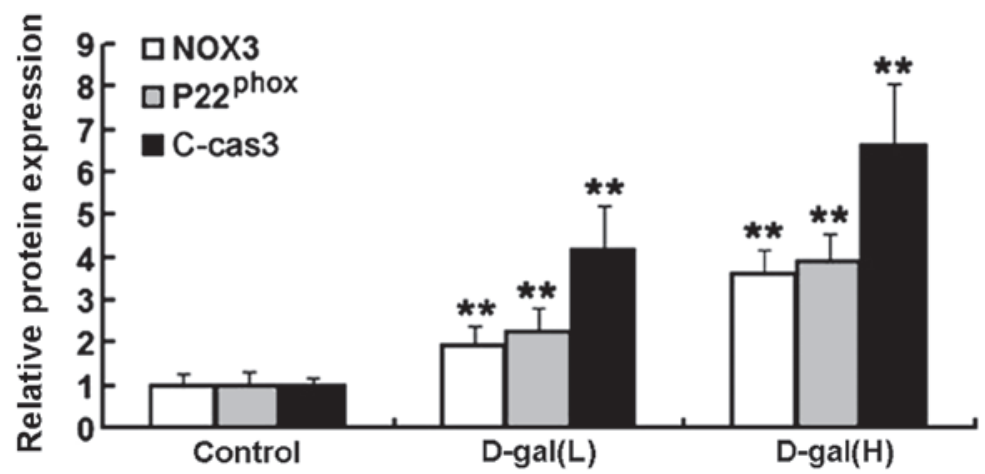

Figure 5. Western blot analysis and densitometric analysis of the expression levels of NOX3, P22 $2^{\text {phox }}$ and C-cas3 in the cochleae. (A) Expression levels of NOX3, P22 $2^{\text {phox }}$ and C-cas3 in the different treatment groups, determined using western blot analysis. (B) Relative protein expression levels of NOX3, P22 $2^{\text {phox }}$ and C-cas 3 were significantly increased in the D-gal groups, compared with the control group. The data are expressed as the mean \pm standard deviation of six rats per group. "** P<0.01, vs. control group. C-cas3, cleaved caspase 3; D-gal, D-galactose; H, 500 mg/kg; L, 150 mg/kg; NOX3, NADPH oxidase 3 .
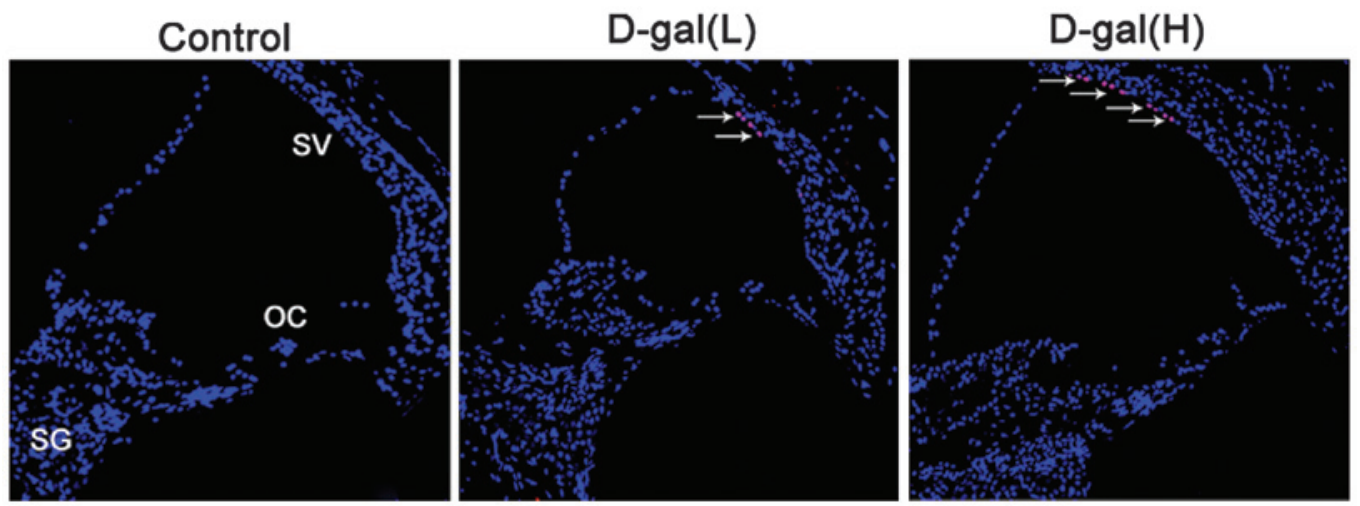

Figure 6. Apoptotic cells in the cochleae of the rats of the different treatment groups. No apoptotic cells were detected in the control group. Apoptotic cells (arrows) were observed in the basal turn of the cochleae of rats from the D-gal groups, determined using terminal deoxynucleotidyl transferase-mediated deoxyuridine triphosphate nick-end-labelling staining. The nuclei were labeled with DAPI. Fluorescent images of the cochleae are presented at x200 magnification. D-gal, D-galactose; H, $500 \mathrm{mg} / \mathrm{kg} ; \mathrm{L}, 150 \mathrm{mg} / \mathrm{kg} ; \mathrm{SV}$, stria vascularis; OC, organ of Corti; SG, spiral ganglion.

Compared with the control group, the protein expression levels of NOX3, P22 $2^{\text {phox }}$ and C-cas3 in the D-gal(L) group increased by 1.92-, 2.25-and 4.18-fold, respectively. The protein expression levels of NOX3, P22 $2^{\text {phox }}$ and C-cas3 in the D-gal $(\mathrm{H})$ group increased by 3.63-, 3.87- and 6.59-fold, respectively (Fig. 5B).

Cell apoptosis is induced by D-gal. To further understand the occurrence of apoptosis induced by D-gal in the cochleae, the numbers of apoptotic cells were determined using TUNEL staining. As shown in Fig. 6, TUNEL-positive cells were located only in the cochleae of the D-gal-treated rats. A small number of TUNEL-positive cells were limited to the SV of the basal turn of the cochleae.

\section{Discussion}

The results of the present study demonstrated that the levels of $\mathrm{H}_{2} \mathrm{O}_{2}$ and MDA increased, and the activity of T-SOD decreased in the blood of rats following 8 weeks of D-gal exposure, which indicated that an animal model of mimetic aging was successfully established by D-gal (40). The results also indicated that the accumulation of mtDNA
CD was significantly increased in the cochleae following treatment with D-gal, which is concordant with the results of previous studies (20-24). Mitochondria are one of the predominant generators of ROS within the cell $(41,42)$. The mitochondrial theory of aging states that ROS generated inside mitochondria damage key mitochondrial components, including mtDNA and respiratory chain complex proteins. This damage accumulates with time and ultimately leads to permanent age-associated mitochondrial dysfunction, which in turn contributes to the aging phenotypes $(43,44)$. The mtDNA 4977 bp deletion in humans, also known as the CD, and the corresponding mtDNA 4834-bp deletion in rats, is the most frequent age-associated mtDNA damage, therefore, $\mathrm{CD}$ has been used as a biomarker for aging $(38,45,46)$. An association between elevated mtDNA CD and presbycusis has been observed in several studies (35,47-49). Although no significant difference is observed in elevation of the auditory brainstem response (ABR) threshold between rats with mtDNA CD induced by D-gal and control rats, the hearing threshold in the rats carrying the mtDNA CD increases significantly following aminoglycoside antibiotic injection, compared with the control rats (20). These results indicate that the mtDNA CD may not directly lead to hearing loss, but 
rather act as a predisposing factor that enhances the sensitivity of the cochleae to aminoglycoside antibiotics (20). To further evaluate D-gal-induced mitochondrial damage in the cochleae, the present study investigated changes in the mitochondrial ultrastructure using TEM. The results indicated that numerous mitochondria were degenerated in different cells of the cochleae in rats following 8 weeks of D-gal exposure. Notably, increased accumulation of mtDNA CD and mitochondrial ultrastructural damage in the cochleae of D-gal-treated rats significantly correlated with increased expression levels of $8-\mathrm{OHdG}$, a biomarker of DNA oxidative damage $(50,51)$. Therefore, these findings suggested that chronic D-gal treatment and the elicited oxidative stress inside mitochondria may contribute to the increased frequency of mtDNA CD and mitochondrial ultrastructural damage in the cochleae of D-gal-treated rats.

The NADPH oxidase system is another important source of ROS production (52). The expression of NOX3 is almost restricted to the cochleae (26), and NOX3-dependent superoxide production is dependent on P22 $2^{\text {phox }}(27)$. Previously, the involvement of NOX3 in cisplatin-induced hearing loss. Knockdown of NOX3 using small interfering (si)RNA inhibited cisplatin ototoxicity, as evidenced by the protection of the outer hair cells from damage, and reduced threshold shifts in ABR in the rat $(29,30)$. Furthermore, transtympanic administration of NOX3 siRNA reduced the expression of B cell lymphoma 2 (Bcl-2)-associated protein $\mathrm{X}$ (Bax), reversed the decreased expression of Bcl-2 and attenuated the apoptosis induced by cisplatin in the cochleae (29). The results of the present study demonstrated that the expression levels of NOX3 and $\mathrm{P} 22^{\text {phox }}$ were significantly increased in the cochleae of rats in the D-gal groups, compared with those in the control group. The overexpression of NOX3 and P22 ${ }^{\text {phox }}$ may partly explain the mitochondrial oxidative damage in the cochleae and the occurrence of apoptosis in the SV of the cochleae, in the rats of the D-gal groups.

Apoptosis was also induced by the accumulation of mtDNA mutations (50). In the mitochondrial signalling pathway of apoptosis, mitochondrial dysfunction can lead to permeabilization of the mitochondrial outer membrane, the release of cytochrome $c$ into the cytosol and the activation of key effector protease, caspase-3, by proteolytic cleavage $(53,54)$. To determine whether increased expression levels of $\mathrm{C}$-cas3 is a feature in the cochleae of D-gal-induced aging rats, soft tissue samples from the cochleae of rats in the treatment groups were examined using western blot analysis. The results indicated that $\mathrm{D}$-gal significantly increased the protein expression levels of $\mathrm{C}$-cas3. Apoptosis is also associated with nuclear DNA fragmentation. The present study examined sections of the cochleae using a TUNEL assay, which detects apoptotic cells in situ. Although a small number of apoptotic cells were located in the SV of the basal turn of the cochleae from the D-gal-induced aging rats, the region of damage in the SV of cochleae may not be sufficient to cause hearing loss (55).

In conclusion, the findings of the present study demonstrated that a marked increase in the expression of NOX3 was involved in the accumulation of mtDNA mutations and in the activation of caspase-3-dependent apoptosis in the cochleae of D-gal-induced aging rats. NOX3 may serve as a useful therapeutic target to prevent or reduce the rate of development of presbycusis.

\section{Acknowledgements}

The present study was supported by funding from the Science and Technology Development Foundation of Shenzhen, China (grant no. JCYJ20140411092351692), the Medical Scientific Research Foundation of Guangdong Province, China (grant no. B2014370) and the Science and Technology Development Foundation of Shenzhen Nanshan District, China (grant no. 2012014).

\section{References}

1. Gates GA and Mills JH: Presbycusis. Lancet 366: 1111-1120, 2005.

2. Yamasoba T, Someya S, Yamada C, Weindruch R, Prolla TA and Tanokura M: Role of mitochondrial dysfunction and mitochondrial DNA mutations in age-related hearing loss. Hear Res 226: 185-193, 2007.

3. Lu J, Zheng YL, Wu DM, Luo L, Sun DX and Shan Q: Ursolic acid ameliorates cognition deficits and attenuates oxidative damage in the brain of senescent mice induced by D-galactose. Biochem Pharmacol 74: 1078-1090, 2007.

4. Zhang ZF, Fan SH, Zheng YL, Lu J, Wu DM, Shan Q and Hu B: Purple sweet potato color attenuates oxidative stress and inflammatory response induced by d-galactose in mouse liver. Food Chem Toxicol 47: 496-501, 2009

5. 5Liu CM, Ma JQ and Lou Y: Chronic administration of troxerutin protects mouse kidney against D-galactose-induced oxidative DNA damage. Food Chem Toxicol 48: 2809-2817, 2010.

6. Chen CF, Lang SY, Zuo PP, Yang N, Wang XQ and Xia C: Effects of D-galactose on the expression of hippocampal peripheral-type benzodiazepine receptor and spatial memory performances in rats. Psychoneuroendocrinology 31: 805-811, 2006.

7. Hua X, Lei M, Zhang Y, Ding J, Han Q, Hu G and Xiao M: Long-term D-galactose injection combined with ovariectomy serves as a new rodent model for Alzheimer's disease. Life Sci 80: 1897-1905, 2007.

8. Kumar A, Prakash A and Dogra S: Naringin alleviates cognitive impairment, mitochondrial dysfunction and oxidative stress induced by D-galactose in mice. Food Chem Toxicol 48: 626-632, 2010.

9. Lu J, Wu DM, Zheng YL, Hu B and Zhang ZF: Purple sweet potato color alleviates D-galactose-induced brain aging in old mice by promoting survival of neurons via PI3K pathway and inhibiting cytochrome C-mediated apoptosis. Brain Pathol 20: 598-612, 2010.

10. Zhang ZF, Lu J, Zheng YL, Hu B, Fan SH, Wu DM, Zheng ZH, Shan Q and Liu CM: Purple sweet potato color protects mouse liver against d-galactose-induced apoptosis via inhibiting caspase-3 activation and enhancing PI3K/Akt pathway. Food Chem Toxicol 48: 2500-2507, 2010.

11. Lei M, Hua X, Xiao M, Ding J, Han Q and Hu G: Impairments of astrocytes are involved in the d-galactose-induced brain aging. Biochem Biophys Res Commun 369: 1082-1087, 2008.

12. Hsieh HM, Wu WM and Hu ML: Soy isoflavones attenuate oxidative stress and improve parameters related to aging and Alzheimer's disease in C57BL/6J mice treated with D-galactose. Food Chem Toxicol 47: 625-632, 2009.

13. Cui X, Wang L, Zuo P, Han Z, Fang Z, Li W and Liu J: D-galactose-caused life shortening in Drosophila melanogaster and Musca domestica is associated with oxidative stress. Biogerontology 5: 317-325, 2004.

14. Wei H, Li L, Song Q, Ai H, Chu J and Li W: Behavioural study of the D-galactose induced aging model in C57BL/6J mice. Behav Brain Res 57: 245-251, 2005.

15. Zhang XL, An LJ, Bao YM, Wang JY and Jiang B: d-galactose administration induces memory loss and energy metabolism disturbance in mice: Protective effects of catalpol. Food Chem Toxicol 46: 2888-2894, 2008.

16. Tian Y, Zou B, Yang L, Xu SF, Yang J, Yao P and Li CM: High molecular weight persimmon tannin ameliorates cognition deficits and attenuates oxidative damage in senescent mice induced by D-galactose. Food Chem Toxicol 49: 1728-1736, 2011. 
17. Deng HB, Cui DP, Jiang JM, Feng YC, Cai NS and Li DD: Inhibiting effects of Achyranthes bidentata polysaccharide and Lycium barbarum polysaccharide on nonenzyme glycation in D-galactose induced mouse aging model. Biomed Environ Sci 16: 267-275, 2003.

18. Deng HB, Cheng CL, Cui DP, Li DD, Cui L and Cai NS: Structural and functional changes of immune system in aging mouse induced by D-galactose. Biomed Environ Sci 19: 432-438, 2006.

19. Uddin MN, Nishio N, Ito S, Suzuki H and Isobe K: Toxic effects of D-galactose on thymus and spleen that resemble aging. J Immunotoxicol 7: 165-173, 2010.

20. Kong WJ, Hu YJ, Wang Q, Wang Y, Han YC, Cheng HM, Kong W and Guan MX: The effect of the mtDNA4834 deletion on hearing. Biochem Biophys Res Commun 344: 425-430, 2006.

21. Kong WJ, Wang Y, Wang Q, Hu YJ, Han YC and Liu J: The relation between D-galactose injection and mitochondrial DNA 4834 bp deletion mutation. Exp Gerontol 41: 628-634, 2006.

22. Peng W, Hu Y, Zhong Y, Chen B, Sun Y, Yang Y and Kong W: Protective roles of alpha-lipoic acid in rat model of mitochondrial DNA4834bp deletion in inner ear. J Huazhong Univ Sci Technolog Med Sci 30: 514-518, 2010.

23. Zhong Y, Hu YJ, Chen B, Peng W, Sun Y, Yang Y, Zhao XY, Fan GR, Huang X and Kong WJ: Mitochondrial transcription factor A overexpression and base excision repair deficiency in the inner ear of rats with D-galactose-induced aging. FEBS J 278: 2500-2510, 2011

24. Zhong Y, Hu YJ, Yang Y, Peng W, Sun Y, Chen B, Huang X and Kong WJ: Contribution of common deletion to total deletion burden in mitochondrial DNA from inner ear of d-galactose-induced aging rats. Mutat Res 712: 11-19, 2011.

25. Bedard K and Krause KH: The NOX family of ROS-generating NADPH oxidases: Physiology and pathophysiology. Physiol Rev 87: 245-313, 2007.

26. Bánfi B, Malgrange B, Knisz J, Steger K, Dubois-Dauphin M and Krause KH: NOX3, a superoxide-generating NADPH oxidase of the inner ear. J Biol Chem 279: 46065-46072, 2004.

27. Ueno N, Takeya R, Miyano K, Kikuchi H and Sumimoto H: The NADPH oxidase Nox3 constitutively produces superoxide in a p22phox-dependent manner: Its regulation by oxidase organizers and activators. J Biol Chem 280: 23328-23339, 2005.

28. Mukherjea D, Whitworth CA, Nandish S, Dunaway GA, Rybak LP and Ramkumar V: Expression of the kidney injury molecule 1 in the rat cochlea and induction by cisplatin. Neuroscience 139: 733-740, 2006.

29. Mukherjea D, Jajoo S, Kaur T, Sheehan KE, Ramkumar V and Rybak LP: Transtympanic administration of short interfering (si)RNA for the NOX3 isoform of NADPH oxidase protects against cisplatin-induced hearing loss in the rat. Antioxid Redox Signal 13: 589-598, 2010

30. Mukherjea D, Jajoo S, Sheehan K, Kaur T, Sheth S, Bunch J, Perro C, Rybak LP and Ramkumar V: NOX3 NADPH oxidase couples transient receptor potential vanilloid 1 to signal transducer and activator of transcription 1-mediated inflammation and hearing loss. Antioxid Redox Signal 14: 999-1010, 2011.

31. Youle RJ and Strasser A: The BCL-2 protein family: Opposing activities that mediate cell death. Nat Rev Mol Cell Biol 9: 47-59, 2008.

32. Someya S, Yamasoba T, Weindruch R, Prolla TA and Tanokura M: Caloric restriction suppresses apoptotic cell death in the mammalian cochlea and leads to prevention of presbycusis. Neurobiol Aging 28: 1613-1622, 2007

33. Someya S, Yamasoba T, Kujoth GC, Pugh TD, Weindruch R, Tanokura $\mathrm{M}$ and Prolla TA: The role of mtDNA mutations in the pathogenesis of age-related hearing loss in mice carrying a mutator DNA polymerase gamma. Neurobiol Aging 29 : 1080-1092, 2008.

34. Yu F, Hao S, Zhao Y, Yang H, Fan XL and Yang J: In utero and lactational $\beta$-carotene supplementation attenuates $\mathrm{D}$-galactose-induced hearing loss in newborn rats. Food Chem Toxicol 49: 1697-1704, 2011.
35. Chen B, Zhong Y, Peng W, Sun Y and Kong WJ: Age-related changes in the central auditory system: Comparison of D-galactose-induced aging rats and naturally aging rats. Brain Res 1344: 43-53, 2010.

36. Chen B, Zhong Y, Peng W, Sun Y, Hu YJ, Yang Y and Kong WJ Increased mitochondrial DNA damage and decreased base excision repair in the auditory cortex of D-galactose-induced aging rats. Mol Biol Rep 38: 3635-3642, 2011

37. Du Z, Yang Y, Hu Y, Sun Y, Zhang S, Peng W, Zhong Y, Huang X and Kong W: A long-term high-fat diet increases oxidative stress, mitochondrial damage and apoptosis in the inner ear of d-galactose-induced aging rats. Hear Res 287: 15-24, 2012.

38. Nicklas JA, Brooks EM, Hunter TC, Single R and Branda RF: Development of a quantitative PCR (TaqMan) assay for relative mitochondrial DNA copy number and the common mitochondrial DNA deletion in the rat. Environ Mol Mutagen 44: 313-320, 2004.

39. Livak KJ and Schmittgen TD: Analysis of relative gene expression data using real-time quantitative PCR and the 2(-Delta Delta C(T)) Method. Methods 25: 402-408, 2001

40. Ho SC, Liu JH and Wu RY: Establishment of the mimetic aging effect in mice caused by D-galactose. Biogerontology 4: 15-18, 2003.

41. Turrens JF: Mitochondrial formation of reactive oxygen species. J Physiol 552: 335-344, 2003.

42. Valko M, Leibfritz D, Moncol J, Cronin MT, Mazur M and Telser J: Free radicals and antioxidants in normal physiological functions and human disease. Int J Biochem Cell Biol 39: 44-84, 2007.

43. Loeb LA, Wallace DC and Martin GM: The mitochondrial theory of aging and its relationship to reactive oxygen species damage and somatic mtDNA mutations. Proc Natl Acad Sci USA 102: 18769-18770, 2005.

44. Hiona A and Leeuwenburgh C: The role of mitochondrial DNA mutations in aging and sarcopenia: Implications for the mitochondrial vicious cycle theory of aging. Exp Gerontol 43: 24-33, 2008.

45. Yowe DL and Ames BN: Quantitation of age-related mitochondrial DNA deletions in rat tissues shows that their pattern of accumulation differs from that of humans. Gene 209: 23-30, 1998.

46. Meissner C, Bruse P, Mohamed SA, Schulz A, Warnk H, Storm T and Oehmichen M: The 4977 bp deletion of mitochondrial DNA in human skeletal muscle, heart and different areas of the brain: a useful biomarker or more. Exp Gerontol 43: 645-652, 2008.

47. Bai U, Seidman MD, Hinojosa R and Quirk WS: Mitochondrial DNA deletions associated with aging and possibly presbycusis: A human archival temporal bone study. Am J Otol 18: 449-453, 1997.

48. Ueda N, Oshima T, Ikeda K, Abe K, Aoki M and Takasaka T: Mitochondrial DNA deletion is a predisposing cause for sensorineural hearing loss. Laryngoscope 108: 580-584, 1998.

49. Markaryan A, Nelson EG and Hinojosa R: Quantification of the mitochondrial DNA common deletion in presbycusis. Laryngoscope 119: 1184-1189, 2009.

50. Kujoth GC, Hiona A, Pugh TD, Someya S, Panzer K, Wohlgemuth SE, Hofer T, Seo AY, Sullivan R, Jobling WA, et al: Mitochondrial DNA mutations, oxidative stress, and apoptosis in mammalian aging. Science 309: 481-484, 2005.

51. Ma Y, Mehta SL, Lu B and Li PA: Deficiency in the inner mitochondrial membrane peptidase 2-like (Immp21) gene increases ischemic brain damage and impairs mitochondrial function. Neurobiol Dis 44: 270-276, 2011.

52. Lambeth JD: Nox enzymes, ROS, and chronic disease: An example of antagonistic pleiotropy. Free Radic Biol Med 43: 332-347, 2007.

53. Hengartner MO: The biochemistry of apoptosis. Nature 407: $770-776,2000$

54. Green DR and Kroemer G: The pathophysiology of mitochondrial cell death. Science 305: 626-629, 2004.

55. Pauler M, Schuknecht HF and White JA: Atrophy of the stria vascularis as a cause of sensorineural hearing loss. Laryngoscope 98: 754-759, 1988. 